\title{
Bioreduction-Mediated Food-Drug Interactions: Opportunities for Oncology Nutrition
}

\author{
Melanie M. Erzinger and Shana J. Sturla*
}

\begin{abstract}
Chemical and biochemical processes underlying food-drug interactions in cancer therapy have not been well addressed with a systematic focus, even though they offer significant potential for enhancing the efficacy of cancer chemotherapy. Bioreductive anticancer drugs are metabolically activated by reductase enzymes. The levels and activities of relevant metabolic enzymes are regulated by transcription factors, which are under the control of chemical interactions with small molecules, including bioactive food components (BFCs) such as minerals, vitamins, and a variety of phytochemicals. One important and well-established process is the upregulation of enzymes involved in xenobiotic metabolism and redox regulation. Thus, BFCs might help to overcome resistances of some cancer cells towards anticancer agents or to increase efficacy by sensitizing cancer cells towards synergistic drugs. By understanding chemical and biochemical processes involved in food-drug interactions, not only can the risk of harmful food-drug interactions be diminished, but appropriate nutritional recommendations for cancer patients can be made and new functional foods with specific benefits in anticancer therapy may be developed.
\end{abstract}

Keywords: Bioreductive drugs · Biotransformation - Cancer therapy · Dietary phytochemicals .

Food-drug interactions

\section{Introduction}

Today, many people use alternative medicine or food supplements to improve their health. In particular, cancer patients undergoing active treatment often seek alternative therapies to try whatever possible to fight their fatal disease. In Switzerland, around $35^{\prime} 000$ people are newly diagnosed with cancer and around 16'000 patients die from it every year. ${ }^{[1]}$ Despite advances in surgical techniques and targeted therapeutics, cancer remains a leading cause of death worldwide.

Shortcomings in cancer therapeutics are due in part to a limited selectivity window for drug responses in malignant cells vs. healthy tissue. Amongst factors that dictate therapeutic success (disease stage, drug selection, genetics, etc.), dietary components or supplements may impact the effect of anticancer agents that have an already narrow therapeutic index, and for

\footnotetext{
${ }^{\star}$ Correspondence: Prof. Dr. S. J. Sturla ETH Zürich

Institute of Food, Nutrition and Health

Schmelzbergstrasse 9

$\mathrm{CH}-8092$ Zürich

Tel.: +41446329175

Fax: +41446321123

E-mail: sturlas@ethz.ch
}

which biotransformation influences drug action and safety. Yet, while drug-drug or radiation-drug interactions in cancer therapy are well studied, food-drug interactions in cancer therapy have barely been addressed and the existing literature is difficult to interpret due to lack of systematic approaches.

Naturally-derived bioactive food components (BFCs), like vitamins, minerals, and phytochemicals, may interfere with the action of drugs, but it is possible that they may do so in a manner that might help overcome resistances of some cancer cells towards anticancer agents, increase selectivity profiles by preferentially protecting normal cells or sensitizing cancer cells, and increase efficacy of chemotherapeutic drugs. BFCs may influence drug action by modulating cellular enzyme activity via direct chemical interactions or by gene-transcription-mediated pathways. A critical gap is in defining the complex array of alterations in cellular profiles, i.e. a quantitative integration of information at the level of chemical reactivity, protein levels, and gene expression, that control drug toxicity as a function of disease progression. A successful outcome could lead to defined diet recommendations for patients that are undergoing a specific cancer therapy and could also result in the development of new functional foods with specific benefits in cancer therapy. This review will focus on possible benefits of enzyme-mediated food-drug interactions and will show dif- ferent classes of BFCs and bioreductive anticancer drugs under current investigation as possible partners in such interactions.

\section{Food Components Stimulate Metabolic Enzymes}

Diet influences a number of critical cellular processes. Examples include regulation of the cell cycle and apoptosis, cell adhesion, cell proliferation, and stress responses, such as redox-regulation and DNA repair. ${ }^{[2-4]}$ Besides these fundamental biological processes, toxic responses to chemicals can be influenced by nutrition, in particular by the large variety and amount of BFCs present in the human diet. For example, the trace mineral selenium (Se) is found in cereals, nuts, vegetables and some meats and seafood. ${ }^{[5]}$ The phytochemicals sulforaphane (SFN, Fig. 1) and phenethyl isothiocyanate (PEITC, Fig. 1) are both isothiocyanates derived from cruciferous vegetables. Especially watercress is a rich source of PEITC, and SFN is most abundant in broccoli. ${ }^{[6]}$ Polyphenols are another group of phytochemicals present in the human diet.[7] Resveratrol (Fig. 1) is a phytoalexin that is found in the skin and seeds of grapes and therefore is also abundant in red wine. Quercetin (Fig. 1) is ubiquitously present in fruits and vegetables and most abundant in onions, apples, tea, broccoli, and red wine. Curcumin (Fig. 
1) can be found in the spice turmeric. ${ }^{[8,9]}$ These are just some prominent examples of BFCs but this list could be expanded due to the huge variety of phytochemicals contained in the human diet. From a cellular perspective, BFCs can influence enzyme activity directly or as inducers of cellular signaling. For example, the compounds presented above are established to induce the activity of transcription factors and thus influence levels of biotransforming and redox-regulating enzymes. ${ }^{[10]}$ A key unanswered question for which current research is underway concerns the differences in induction responses in cancer or healthy cells. ${ }^{[11]}$

In cancer cells, metabolic processes are disrupted compared to normal cells. The widely established fact that cancer cells deviate from redox homeostasis may be considered in at least three different, but interrelated, models. First, cancer cells are hypoxic; their reduction of dissolved oxygen (i.e. low oxygen pressure/tension) has been recognized for decades. ${ }^{[12]}$ Hypoxia is associated with a worsened disease prognosis and aggressive phenotypes. It results in the induction of transcription factors such as hypoxia inducible factor-1 (HIF-1), which mediates adaptive responses underlying enhanced survival under hypoxic conditions. However, the altered metabolic capacities and redox status also offer a vulnerability of cancer over normal cells in that cancer cells may be selectively targeted by redox-sensitive drugs.[13] A second consequence of the cancer phenotype is increased reactive oxygen species (ROS), such as superoxide, hydroxyl radical, and nitric oxide, detected in almost all types of cancers. ${ }^{[14]}$ ROS are toxicologically important because of their direct chemical reactivity and because they mediate ROS-sensitive signaling pathways and cellular functions. Examples include chemical reaction with cellular molecules, such as DNA, protein, lipids, or metabolites, and other small molecules including drugs. Furthermore, ROS and their reactions influence kinaseregulated signaling pathways, and cell proliferation, motility and metastasis. Finally, a third perspective on tumorigenic deviation from redox homeostasis is that expression levels of particular enzymes may be altered or uniquely susceptible to transcriptional activation triggers. Examples include increases in the levels of drug-metabolizing enzymes or other redox-regulating reductase enzymes. Specific examples pertaining to this concept, including how alterations in enzyme levels impact the toxicity of bioreductive drugs, are the main focus of this review.

Many known, and surely as yet unknown, food components comprising a wide structural range of chemicals influ-

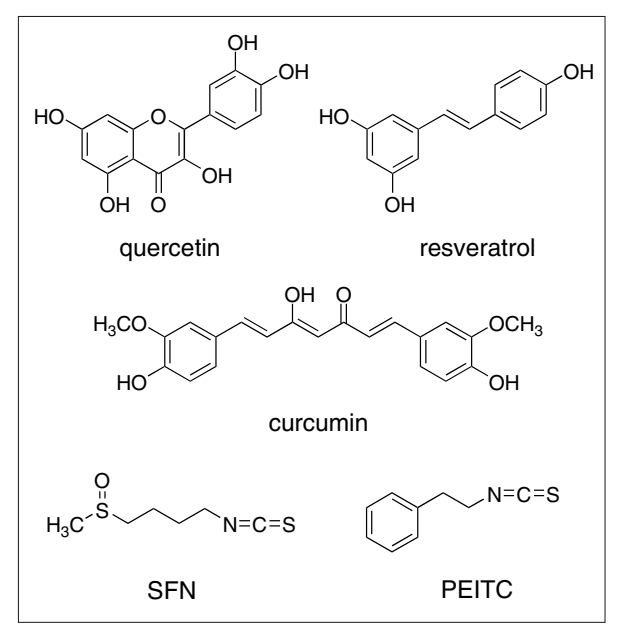

Fig. 1. Five examples of BFCs that interact with the Nrf2-Keap1 pathway: quercetin, resveratrol, curcumin, sulforaphane (SFN), and phenethyl isothiocyanate (PEITC).

ence metabolic enzyme levels by transcriptional activation. A common underlying mechanism involves the Nrf2-Keap1 transcriptional regulation pathway. ${ }^{[15]}$ This pathway, which is also termed the antioxidant response pathway, is responsible for the maintenance of a favorable balance between bioactivation and detoxification of endogenous and exogenous reactive species via upregulation of a variety of antioxidative and phase 2 enzymes. ${ }^{[16]} \mathrm{A}$ DNA promoter region consensus sequence called the antioxidant response element (ARE) is common to genes controlled by the Nrf2 transcription factor. Under basal conditions Nrf2 is bound to Keap1, which is a cysteine-rich protein in the cytoplasm and therefore a good sensor of oxidative and electrophilic stress. ${ }^{[17]}$ Most ARE inducers contain thiol-reactive electrophilic moieties and therefore they can directly bind to Keap1. ${ }^{[18]}$ But they can also change the redox potential in the cell, e.g. by interacting with glutathione and altering the intracellular GSH:GSSG, ratio or by the formation of ROS. Both ways provoke the release of Nrf2, its phosphorylation, and translocation into the nucleus. In the nucleus, Nrf2 binds to the ARE of target genes, triggering transcription.

To identify functional gene clusters that are regulated by $\mathrm{Nrf} 2$, comparative analyses of global gene expression changes in wildtype and $\mathrm{Nrf2}$-null mice treated with known Nrf2 inducers have been performed. ${ }^{[19,20]}$ For example, Thimmulappa et al. showed that 26 genes were upregulated by SFN treatment in an Nrf2-dependent manner in the small intestine of mice. ${ }^{[19]}$ Most of these genes were for enzymes associated with glutathione synthesis and NADPH generation, or for detoxification enzymes, such as glutathione $S$-transferases (GSTs) and NAD(P)H:quinone oxidoreductase-1 (NQO1). Kong and coworkers showed that
$12 \mathrm{~h}$ after a single dose of SFN in wild-type mice, the expression of 2353 hepatic genes was increased in an Nrf2-dependent manner, and that these Nrf2-controlled genes represented $70 \%$ of the total $\mathrm{SFN}$-inducible genes. ${ }^{[20]}$ The corresponding gene products were stress response proteins, xenobiotic metabolizing enzymes, antioxidant proteins, transporters, growth arrest-related proteins, and transcription factors. Similar results were obtained when PEITC was used as an Nrf2 inducer.[21]

In addition to isothiocyanates, another group of interesting dietary phytochemicals that interfere with the Nrf2-Keap1 pathway are the polyphenols. Quercetin induces ARE-mediated NQO1 expression via upregulation of Nrf2 and repression of Keap1 in human liver cells in vitro. ${ }^{[22]}$ Curcumin is chemopreventive in mice at dietary levels most likely by increasing nucleosolic Nrf2 levels, which triggers the expression of GST isoforms and NQO1 in various tissues.[23] Resveratrol induces, by activation of $\mathrm{Nrf} 2$, glutathione synthesis that protects against cigarette smoke-mediated oxidative stress in human lung epithelial cells. ${ }^{[24]}$

$\mathrm{Se}$ is an essential micronutrient due to its incorporation into selenoproteins in the form of selenocystein. These selenoenzymes play an important role in cellular antioxidant systems ${ }^{[25]}$ and changes in their levels may have an impact on cellular processes, including redox-regulating enzyme activity. Additionally, some Se compounds, such as $S e$-allylselenocysteine, dimethyl diselenide, and methaneselenol, have been shown to induce phase 2 enzymes via the Nrf2-Keap1 pathway. ${ }^{[26]}$

\section{Cancer Drug Toxicity Control by Metabolic Enzymes}

Chemotherapy drugs may act directly on cellular targets or they may require metabolic activation to exert their cytotoxic or cytostatic effects. Thus, metabolic changes triggered within a cell may significantly influence drug activity. As such, activation of a detoxifying transformation may eliminate a direct-acting drug, but on the other hand activate a pro-drug. Bioreductive drugs rely on metabolic activation by enzyme-catalyzed reduction. ${ }^{[27]}$ Many bioreductive drugs can be divided into four structural sub-groups, namely quinones, nitroaromatics, N-oxides, and metal complexes. ${ }^{[28]}$ Fig. 2 shows examples for the first three sub-groups.

A prototype quinone-containing bioreductive agent, mitomycin C (MMC, Fig. 2) is an antitumor antibiotic discovered in the 1950 s that is used to treat several types of cancer, such as gastric, breast, and bladder cancer. ${ }^{[29]}$ Later it was recognized that the bioreduction and the activation of MMC 


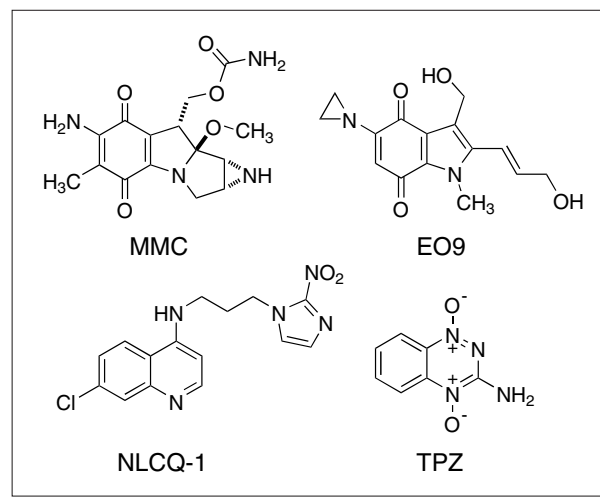

Fig. 2. Examples of structural sub-groups of bioreductive anticancer prodrugs: quinones (MMC, EO9), nitroaromatics (NLCQ-1), and $\mathrm{N}$-oxides (TPZ).

could be facilitated by reducing enzymes in cancer cells. It has been suggested that the major cause for activated MMC's antitumor effect are DNA crosslinks that inhibit DNA synthesis. ${ }^{[29]}$ However, many questions regarding its activity remain unanswered and under investigation. For example, a recent study suggested the involvement of mitochondrial DNA damage in MMC activity. ${ }^{[30]}$

The chemical transformations involved in MMC bioreduction and its reaction with DNA are well-established (Scheme 1). Under anaerobic conditions MMC can be reduced by a one- or two-electron process, and subsequent spontaneous loss of methanol leads to the formation of an unstable reactive intermediate hydroquinone. ${ }^{[29]} \mathrm{Re}$ arrangement of the hydroquinone followed by nucleophilic addition of DNA, yields a mono-alkylated DNA-MMC adduct. Intramolecular displacement of the carbamate group from the mono-adduct finally results in the DNA-MMC-DNA crosslinked adduct 1. ${ }^{[29]}$ Different enzymes may be involved in the reductive activation of MMC. Examples are NQO1, NADPH/cytochrome P450 reductase, NADPH-cytochrome $\mathrm{C}$ reductase, $\mathrm{xanthine-oxidase,} \mathrm{and}$ some flavoprotein transhydrogenases. [29] After the identification of MMC as a bioreductive anticancer drug, the search for analogues with greater selectivity toward reducing cancer cells was initiated. For example, a series of synthetic indolequinones have been medicinally evaluated and one analogue, EO9 (Fig. 2) showed superior efficacy and advanced in clinical trials. [31]

The nitroaromatics, such as NLCQ-1 (Fig. 2), are a second group of bioreductive drugs. They are reduced via the stepwise addition of up to six electrons catalyzed by various one-electron reductases and exhibit weak DNA-intercalating ability. [32] The nitroradical anion (one-electron addition), the nitroso $\left(2 \mathrm{e}^{-}\right)$, and the hydroxylamine $\left(4 \mathrm{e}^{-}\right)$products are the most important toxic metabolites in their reductive pathway and under hypoxic conditions the nitroso- and hydroxylamine, or their ring-cleavage products, such as glyoxal, lead to covalent reaction products. ${ }^{[32]}$

Tirapazamine (TPZ, Fig. 2), a bioreductive agent in current clinical trials, belongs to the third sub-group, the $\mathrm{N}$-oxides. The cytotoxicity of TPZ increases with diminishing oxygen levels. ${ }^{[33]}$ This makes TPZ an interesting drug for treating, at intermediate oxygen tension, cells resistant to radiotherapy but not sufficiently hypoxic to be a target for other bioreductive drugs. The activation of TPZ is catalyzed by classical one-electron reductases, cytochrome $\mathrm{P} 450$ and cytochrome $\mathrm{P} 450$ reductase, and the resulting one-electron reduction product was hypothesized to mediate its cytotoxicity. This nitroxide radical intermediate is reactive; in the presence of molecular oxygen it can be back-oxidized, i.e. neutralized, and in the absence of oxygen it forms an oxidizing radical that can cause DNA damage. ${ }^{[13]}$

A fourth group of bioreductive drugs that select for tumor hypoxia are metal complexes, particularly those of cobalt. The mechanism of reductive activation of metal complexes is still controversial. It is suggested that redox cycling between oxidation states of the metal complex in the presence of oxygen is the reason for their hypoxia-selectivity. ${ }^{[28]}$

Acylfulvenes (AFs) are under investigation as a unique type of bioreductive anticancer agents. They are semi-synthetic derivatives of the natural sesquiterpenes illudin $\mathrm{S}$ and $\mathrm{M}$, mycotoxins produced by the Jack O'Lantern mushroom Omphalotus illudins (Fig. 3). ${ }^{[34,35]}$ Illudins are cytotoxic, but exhibit low selectivity towards cancer cells compared to normal cells. Thus, they have a narrow therapeutic window, meaning that the therapeutic dose and the toxic dose of the drug are similar. ${ }^{[36]}$ AFs, in contrast, show improved selectivity towards cancer cells over normal cells, ${ }^{[37]}$ and one analogue, hydroxymethylacylfulvene (HMAF), has been evaluated in extensive preclinical and advanced clinical investigations. ${ }^{[37,38]}$
Cytosolic bioreduction and hydrolysis are involved in transforming AF to the major metabolite 2 (Scheme 2). ${ }^{[35]}$ In 2004, Kensler and co-workers demonstrated that the cytosolic enone reductase alkenal/one oxidoreductase (AOR), which is identical to a splice variant of prostaglandin reductase 1 (PTGR1), catalyzes the reduction of AFs in a NADPH-dependent two-electron process. ${ }^{[39]}$ AOR is a cytosolic enzyme that catalyzes the reductive deactivation of many electrophilic substrates, like lipid peroxidation products, to prevent biomolecular adduct formation involving the carboncarbon double bond of $\alpha, \beta$-unsaturated ketones and aldehydes. ${ }^{[40]}$ In the absence of enzymatic activation, AFs alkylate DNA by direct conjugate addition. ${ }^{[11]}$ Additionally, AFs interact with and inhibit glutathione reductase, consistent with the potential for direct interactions with cytosolic redox-regulating enzyme interactions to be involved in the cellular response to the drugs. ${ }^{[42-44]}$ In the presence of AOR, however, the efficiency of DNA alkylation is increased over a hundred-fold and the reaction with DNA instead involves the cyclopropyl group. ${ }^{[41,45]}$ The kinetic parameters for AOR-mediated activation of AF analogues and their cytotoxicities are positively correlated; the highest toxicities were observed in cells engineered to overexpress AOR and for analogues that were efficiently activated by the enzyme. ${ }^{46]}$

When AF is enzymatically reduced, the resulting intermediate is unstable (indeed, it has never been isolated) and quickly reacts with nucleophiles, including DNA (Scheme 2). ${ }^{[41,45]}$ The most abundant DNA alkylation product arises from alkylation of adenosine to yield 3-AF-Adn. This adduct is unstable and depurinates with a half-life of $8.5 \mathrm{~h}$ in naked cell-free DNA. ${ }^{[41]}$ In recent studies, a stable yet chemically reduced analogue of $\mathrm{AF}$ has been synthesized. ${ }^{[44]}$ It was shown that the reaction of this analogue with DNA gives rise to alkylation profiles in DNA that are the same as generated during the AOR-catalyzed reaction, and that the cytotoxicity of the stable reduced form does not depend on AOR levels as it does for AF. ${ }^{[4]}$ Therefore, this ana-

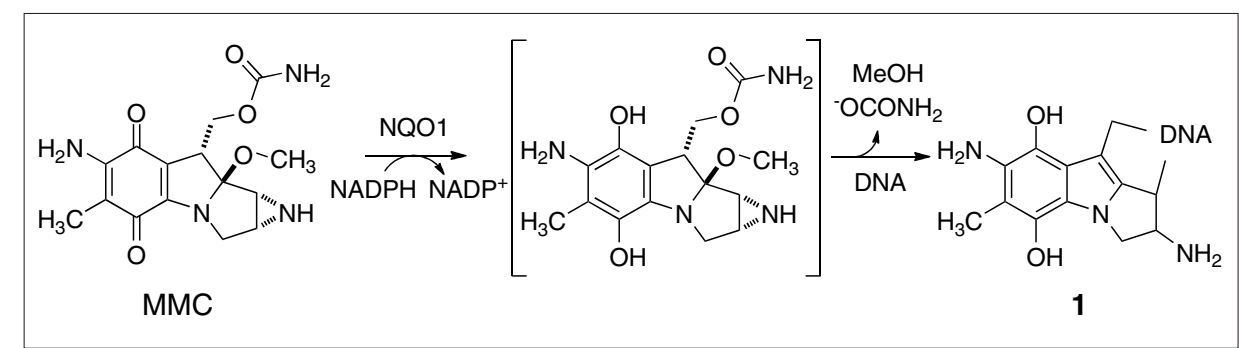

Scheme 1. Reductive activation of MMC. The formation of the DNA-crosslink $\mathbf{1}$ is a multiple step process, including the loss of methanol and the carbamate. NQO1 is one example of a reductase involved in the reduction of MMC, and others may include NADPH/cytochrome P450 reductase, $\mathrm{NADPH}$-cytochrome $\mathrm{C}$ reductase, xanthine-oxidase, and some flavoprotein transhydrogenases. 


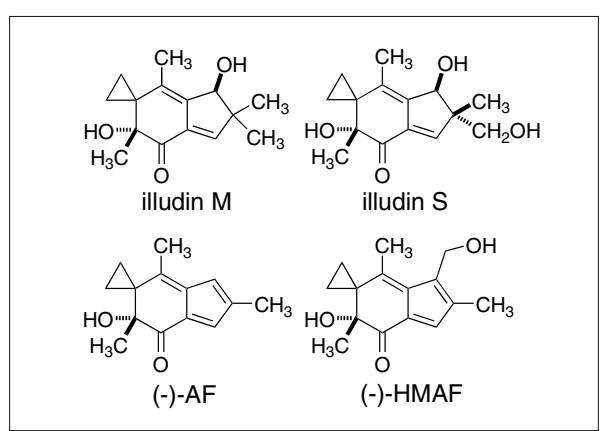

Fig. 3. Natural products, illudin $M$ and $S$, and semisynthetic analogues, AF and HMAF.

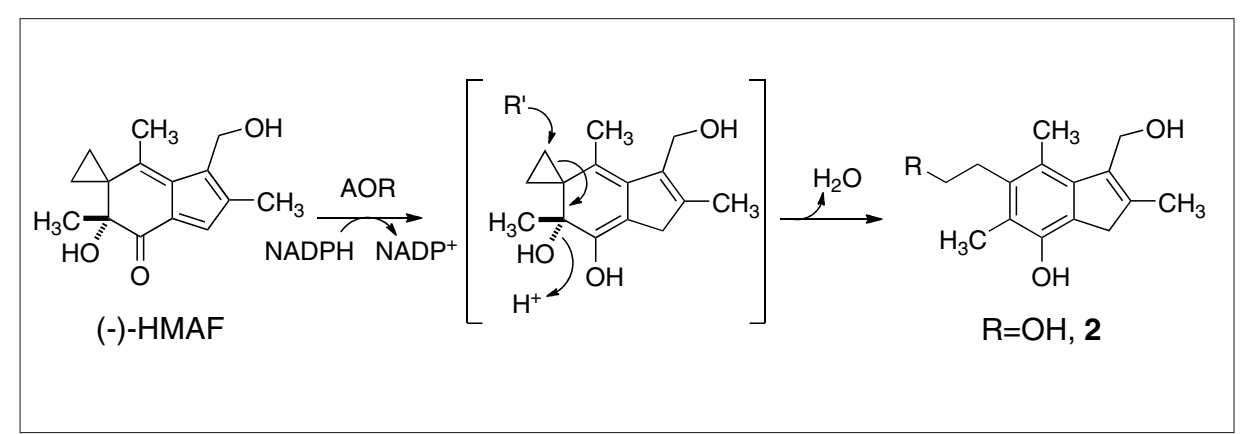

Scheme 2. Proposed mechanism of biomolecular metabolite or adduct formation by HMAF. R' = cellular nucleophiles, such as $\mathrm{H}_{2} \mathrm{O}, \mathrm{GSH}$, DNA, protein; $\mathrm{R}$ = corresponding adducted target.

potentiates the antitumor effects of radiation therapy by suppressing the transcription factor nuclear factor $\kappa \mathrm{B}(\mathrm{NF}-\kappa \mathrm{B})$ and $\mathrm{NF}-\kappa \mathrm{B}-$ regulated gene products, resulting in inhibition of proliferation and angiogenesis. ${ }^{[50]}$ Despite many unanswered issues concerning the mode of action, these studies have been instrumental in guiding recent and ongoing clinical trials. ${ }^{[51,52]}$ Recently another BFC, namely SFN, was shown to enhance radiosensitivity in $\mathrm{HeLa}$ human cervix carcinoma cells by inhibiting DNA double-strand break repair.[53] These examples indicate the potential support of cancer therapy by BFCs.

A connection between $\mathrm{Se}$ and cancer therapy enhancement has previously been suggested by several studies showing that $\mathrm{Se}$ is a highly effective modulator of the therapeutic efficacy and selectivity of anticancer drugs. ${ }^{[54-59]}$ For example, non-toxic doses of Se in the form of methylselenocysteine (MSC) enhanced the efficacy and selectivity of irinotecan against several human tumor xenografts. ${ }^{[55]}$ Inhibition of HIF-1 $\alpha$ by MSC was suggested as the sensitization mechanism. ${ }^{[59]}$ Similarly, MCF-7 breast cancer cells were sensitized to doxorubicininduced apoptosis by pre-conditioning with Se. ${ }^{[56]}$ This sensitization was suggested to be achieved by damping doxorubicin-induced activation of Akt, a protein kinase involved in the regulation of essential cellular functions. A combination of MSC and tamoxifen synergistically inhibited tumor growth in MCF-7 breast cancer xenografts compared to either agent alone. ${ }^{[58]}$ In this case, a decrease in estrogen receptor- $\alpha(\mathrm{ER} \alpha)$ protein, ER $\alpha$ signaling, proliferation and angiogenesis, and increases in tumor cell apoptosis were observed. It was suggested, therefore, that tumor growth inhibition was due, at least in part, to down-regulation of ER $\alpha .{ }^{[58]}$ Minimally toxic concentrations of the organic Se compound methylseleninic acid increased the cytotoxicity of different chemotherapeutic agents, including doxorubicin, etoposide, and 4-hydroxycyclophosphamide, in human B-cell lymphoma cell lines by inhibiting the transcription factor $\mathrm{NF}-\kappa \mathrm{B} .{ }^{[57]}$
Se in the form of selenocysteine is an essential component of some major redoxregulating enzymes, including glutathione peroxidase and thioredoxin reductase (TrxR). A recent study in our lab addressed the effect of selenite on cell sensitivity toward the bioreductive anticancer drugs AF and illudin. ${ }^{[43]}$ After three days of culture in media containing selenite, TrxR levels in HeLa cells were elevated. When cellular TrxR protein levels, as well as TrxR activity, increased four-fold, cells were $50 \%$ more sensitive toward AFs, but not towards illudin S. ${ }^{[43]}$ These data suggest TrxR as a potential additional cytotoxicity target for AFs but not for illudin S. ${ }^{[43]}$ These data support the hypothesis that pre-conditioning with sub-toxic BFC doses may enhance enzyme expression levels and activities, and sensitize cells to anticancer drugs.

The hypothesis that BFC pre-conditioning may enhance drug action is formulated on the basis of examples mentioned above, however, far from being definitely demonstrated, there are a number of complicating chemical and biological factors that must be sorted. While there is limited information regarding the mode of specific BFC-drug interactions, by contrast information regarding BFCmediated xenobiotic metabolism in the context of cancer chemoprevention is extensive, and the underlying chemical and biological processes are closely related. Thus, chemoprevention research could provide hints regarding cellular responses to BFCs that may be invoked in the sensitization of cells toward bioreductive anticancer agents. ${ }^{[10,11,18,60]}$ While a major gap between these frameworks concerns differences in BFC responses between tumor and normal cells, there are many examples of such differences, including large amount of chemoprevention-oriented studies that have been performed with cancer cells. ${ }^{[11]}$

Clearly, enzyme-mediated food-drug interactions are complicated and the ultimate impact and potential improvements in therapy remain elusive. Together with defining differences between tumor and normal cells, further challenges to be adgrowth factors (EGFR, IGF-1R) signaling pathways. In colorectal cancer, curcumin 
dressed concern the multiplicity of enzymes that may be regulated by a BFC, as well as the potential influence of a network of small cellular changes for which it is technically difficult to derive statistically significant data. A goal is an enhanced understanding of the relationship between food components, gene transcription, protein expression, metabolism, and cytotoxicity, and how these relationships may change with disease progression. Thus, a system-wide analysis involving modern analytical platforms and close cross-disciplinary exchange between fundamental chemistry, biochemistry, and molecular biology investigations with new technologies and translational strategies, are needed. ${ }^{[61]}$ The fundamental information gained and experimental tools developed by this system-wide analysis could be further leveraged by the food industry and clinicians in support of oncology nutrition.

\section{Conclusion and Future Outlook}

Impact on drug efficacy by a cancer patient's diet or nutritional supplements is emerging as a potentially important, but poorly understood, influence on treatment outcomes, particularly as there is an increasing trend toward oral delivery of anticancer drugs and self-medication with dietary supplements. Knowledge regarding chemistry of the activation of bioreductive anticancer drugs, identification of metabolic enzymes involved in this bioactivation, and information regarding their nutritional regulation suggests that foods containing minerals, vitamins and phytochemicals may significantly impact drug cytotoxicity. There is potential for new functional foods that optimize interaction between current foods and current drugs, or to determine novel functionalities of both old and new food ingredients, while addressing multiple aspects of nutritional challenges faced by the cancer patient.

A better understanding of food-drug interactions in cancer therapy offers the possibility to establish specific dietary guidelines and recommend special diets, suitable foods, or supplements to cancer patients that not only aim at preventing weight loss or overcoming drug side effects, but suit their situation the best by increasing the efficacy of their cancer treatment. This review focused on the potential positive interactions of foods or supplements with cancer treatment, but it has to be emphasized here that there is also the possibility of harmful interactions, especially by taking over-the-counter dietary and herbal supplements. Ultimately, therefore, a better understanding of positive food-drug interactions might also support information provided to cancer patients about food or dietary supplement safety to avoid potential drug resistance or side effects. Finally, a systematic analysis and establishment of a scientific framework underlying food-drug interactions in cancer therapy can open new opportunities for food and nutrition research in academia and industry with the aim of improving cancer therapy.

\section{Acknowledgment}

We thank Dr. Katrin Hecht for helpful discussions, contributions, and editorial critique. We acknowledge the US National Cancer Institute for financial support (RO1CA123007).

Received: April 2, 2011

[1] C. Bouchardy, J.-M. Lutz, C. Kühni, 'Cancer in Switzerland - Situation and development from 1983 to 2007', Swiss Statistics, Swiss Federal Statistical Office: Neuchâtel, 2011.

[2] D. Yu, E. Sekine-Suzuki, L. Xue, A. Fujimori, N. Kubota, R. Okayasu, Int. J. Cancer 2009, $125,1205$.

[3] S. Rajamanickam, R. Agarwal, Drug Dev. Res. 2008, 69, 460 .

[4] B. B. Aggarwal, S. Shishodia, Biochem. Pharmacol. 2006, 71, 1397.

[5] S. J. Fairweather-Tait, R. Collings, R. Hurst, Am. J. Clin. Nutr. 2010, 91, 1484S.

[6] J. W. Fahey, A. T. Zalcmann, P. Talalay, Phytochemistry 2001, 56, 5 .

[7] L. Bravo, Nutr. Rev. 1998, 56, 317

[8] J. Peterson, J. Dwyer, Nutr. Res. 1998, 18, 1995.

[9] N. Khan, F. Afaq, H. Mukhtar, Antioxid. Redox Signaling 2008, 10, 475.

[10] J. K. Kundu, Y.-J. Surh, Phytochem. Rev. 2009, $8,333$.

[11] S. Nair, W. Li, A.-N. T. Kong, Acta Pharmacol. Sin. 2007, 28, 459.

[12] A. L. Harris, Nat. Rev. Cancer 2002, 2, 38

[13] G. T. Wondrak, Antioxid. Redox Signaling 2009, 11, 3013

[14] G.-Y. Liou, P. Storz, Free Radical Res. 2010, $44,479$.

[15] J. D. Hayes, M. McMahon, S. Chowdhry, A. T. Dinkova-Kostova, Antioxid. Redox Signaling 2010, 13, 1713

[16] I. M. Copple, C. E. Goldring, N. R. Kitteringham, B. K. Park, in 'Adverse Drug Reactions', Ed. J. Uetrecht, Springer, Berlin Heidelberg, 2010, p. 233.

[17] T. Primiano, T. R. Sutter, T. W. Kensler, $A d v$. Pharmacol. 1997, 38, 293.

[18] A. L. Eggler, K. A. Gay, A. D. Mesecar, Mol. Nutr. Food Res. 2008, 52, S84.

[19] R. K. Thimmulappa, K. H. Mai, S. Srisuma, T. W. Kensler, M. Yamamoto, S. Biswal, Cancer Res. 2002, 62, 5196.

[20] R. Hu, C. Xu, G. Shen, M. R. Jain, T. O. Khor, A. Gopalkrishnan, W. Lin, B. Reddy, J. Y. Chan, A.-N. T. Kong, Cancer Lett. 2006, 243, 170.

[21] R. Hu, C. Xu, G. Shen, M. R. Jain, T. O. Khor, A. Gopalkrishnan, W. Lin, B. Reddy, J. Y. Chan, A.-N. T. Kong, Life Sci. 2006, 79, 1944.

[22] S. Tanigawa, M. Fujii, D.-X. Hou, Free Radical Biol. Med. 2007, 42, 1690.

[23] R. Garg, S. Gupta, G. B. Maru, Carcinogenesis 2008, 29, 1022

[24] A. Kode, S. Rajendrasozhan, S. Caito, S.-R. Yang, I. L. Megson, I. Rahman, Am. J. Physiol. Lung Cell Mol. Physiol. 2008, 294, L478.

[25] F. P. Bellinger, A. V. Raman, M. A. Reeves, M. J. Berry, Biochem. J. 2009, 422, 11.

[26] R. Brigelius-Flohé, Chem. Biodiv. 2008, 5, 389.
[27] P. Workman, I. J. Stratford, Cancer Metastasis Rev. 1993, 12, 73 .

[28] Y. Chen, L. Hu, Med. Res. Rev. 2009, 29, 29.

[29] J. Verweij, H. M. Pinedo, Anti-Cancer Drugs 1990, 1,5 .

[30] R. G. Snodgrass, A. C. Collier, A. E. Coon, C. A. Pritsos, J. Biol. Chem. 2010, 285, 19068

[31] S. R. McKeown, R. L. Cowen, K. J. Williams, Clin. Oncol. 2007, 19, 427.

[32] M. V. Papadopoulou, W. D. Bloomer, Clin Cancer Res. 2003, 9, 5714.

[33] C. J. Koch, Cancer Res. 1993, 53, 3992.

[34] T. C. McMorris, M. Anchel, J. Am. Chem. Soc. 1965, 87, 1594.

[35] T. C. McMorris, M. J. Kelner, W. Wang, M. A. Diaz, L. A. Estes, R. Taetle, Experientia 1996, 52,75 .

[36] M. J. Kelner, T. C. McMorris, R. Taetle, J. Natl. Cancer Inst. 1990, 82, 1562

[37] J. R. MacDonald, C. C. Muscoplat, D. L. Dexter, G. L. Mangold, S.-F. Chen, M. J. Kelner, T. C. McMorris, D. D. Von Hoff, Cancer Res. 1997, $57,279$.

[38] M. Tanasova, S. J. Sturla, 2011, submitted.

[39] R. A. Dick, X. Yu, T. W. Kensler, Clin. Cancer Res. 2004, 10, 1492

[40] R. A. Dick, M.-K. Kwak, T. R. Sutter, T. W. Kensler, J. Biol. Chem. 2001, 276, 40803.

[41] J. C. Gong, V. G. Vaidyanathan, X. Yu, T. W. Kensler, L. A. Peterson, S. J. Sturla, J. Am. Chem. Soc. 2007, 129, 2101

[42] X. D. Liu, S. J. Sturla, Mol. Biosyst. 2009, 5, 1013.

[43] X. Liu, K. E. Pietsch, S. J. Sturla, Chem. Res. Toxicol. 2011, 24, 726

[44] K. E. Pietsch, J. F. Neels, J. Gong, X. Yu, S. J. Sturla, 2011, submitted.

[45] J. F. Neels, J. Gong, X. Yu, S. J. Sturla, Chem. Res. Toxicol. 2007, 20, 1513 .

[46] J. C. Gong, J. F. Neels, X. Yu, T. W. Kensler, L. A. Peterson, S. J. Sturla, J. Med. Chem. 2006, 49, 2593.

[47] B. B. Patel, A. P. N. Majumdar, Nutr. Cancer 2009, 61, 842 .

[48] J. Y. Koo, H. J. Kim, K.-O. Jung, K.-Y. Park, $J$. Med. Food 2004, 7, 117

[49] B. B. Patel, R. Sengupta, S. Qazi, H. Vachhani, Y. Yu, A. K. Rishi, A. P. N. Majumdar, Int. J. Cancer 2008, 122, 267.

[50] A. B. Kunnumakkara, P. Diagaradjane, S. Guha A. Deorukhkar, S. Shentu, B. B. Aggarwal, S. Krishnan, Clin. Cancer Res. 2008, 14, 2128.

[51] R. Epelbaum, M. Schaffer, B. Vizel, V Badmaev, G. Bar-Sela, Nutr. Cancer 2010, 62, 1137.

[52] G. Bar-Sela, R. Epelbaum, M. Schaffer, Curr. Med. Chem. 2010, 17, 190

[53] D. Yu, E. Sekine-Suzuki, L. Xue, A. Fujimori, N. Kubota, R. Okayasu, Int. J. Cancer 2009, 125, 1205.

[54] Y.-J. Kim, S.-H. Baek, P. N. Bogner, C. Ip, Y. M Rustum, M. G. Fakih, N. Ramnath, Y.-M. Park, J. Cancer Mol. 2007, 3, 37 .

[55] S. Cao, F. A. Durrani, Y. M. Rustum, Clin. Cancer Res. 2004, 10, 2561.

[56] S. Li, Y. Zhou, R. Wang, H. Zhang, Y. Dong, C. Ip, Mol. Cancer Ther. 2007, 6, 1031.

[57] S. Jüliger, H. Goenaga-Infante, T. A. Lister, J. Fitzgibbon, S. P. Joel, Cancer Res. 2007, 67, 10984.

[58] Z. Li, L. Carrier, A. Belame, A. Thiyagarajah, V. A. Salvo, M. E. Burow, B. G. Rowan, Breast Cancer Res. Treat. 2009, 118, 33 .

[59] S. Chintala, K. Tóth, S. Cao, F. A. Durrani, M. M. Vaughan, R. L. Jensen, Y. M. Rustum, Cancer Chemother. Pharmacol. 2010, 66, 899.

[60] M.-K. Kwak, T. W. Kensler, Toxicol. Appl. Pharmacol. 2010, 244, 66.

[61] A. T. Jacobs, L. J. Marnett, Acc. Chem. Res. 2010, 43,673 . 